\title{
ON CERTAIN SUBGROUPS OF A JOIN OF SUBNORMAL SUBGROUPS
}

\author{
by HOWARD SMITH
}

(Received 18 November, 1982)

1. Introduction. Suppose the group $G$ is generated by subnormal subgroups $H$ and $K$, and that $A, B$ are normal subgroups of finite index in $H, K$ respectively. The following question has been asked by $\mathrm{J}$. C. Lennox: Under what circumstances is the subgroup $J=\langle A, B\rangle$ subnormal in $G$ ? In particular, it is of interest to know when $J$ has finite index in $G$, for, if this is the case, we may factor out by the normal core of $J$ in $G$ and apply Wielandt's theorem on joins of subnormal subgroups of finite groups [11] to deduce that $J$ is subnormal in $G$. Here we prove the following result.

Theorem 1. Let $G=\langle H, K\rangle$, with $H, K$ subnormal in $G$, and suppose that $A, B$ are normal subgroups of finite index in $H, K$ respectively. Then, if $G / G^{\prime}$ has finite rank, $J=\langle A, B\rangle$ has finite index in $G$.

It is not possible to dispense with either the hypothesis of finite rank or that of subnormality: If $W=E$ wr $C$ denotes the wreath product of an infinite, elementary abelian $p$-group $E$ by a cyclic group $C$ of order $p$, then $W$ is nilpotent [2] and so $C$ is subnormal in $W$, but $E$ does not have finite index in $W$.

If, on the other hand, $Z=H$ wr $C$, where $H$ is infinite cyclic, then $H$ does not have finite index in $Z$ although $Z$ has rank $p+1$.

Further, it is not difficult to find an example of a group $G^{*}$ satisfying all of the hypotheses of Theorem 1 apart from that of finite rank, such that the resulting join is not even subnormal in $G^{*}$. The group $G$ constructed by P. Hall and described in detail in Theorem 6.1 of [5] is a split extension $M] J$, where $J=\langle H, K\rangle$ is a self-normalising subgroup of $G$ generated by abelian subgroups $H$ and $K$, each subnormal in $G$, and $M=A \times B$ is an elementary abelian 2-group, where $[A, H]=[B, K]=1$. If $x$ is any element of $A$, then $H$ has index 2 in $\langle x\rangle H=L$, say, which is subnormal in $G$ since $H A$ is. However, $J$ is a proper, self-normalising subgroup of $\langle L, K\rangle$. Thus we have proved the following theorem.

THEOREM 2. There is a group $G^{*}$ generated by subnormal abelian subgroups $L$ and $K$, such that $L$ has a subgroup $H$ of index 2 and $\langle H, K\rangle$ is not subnormal in $G^{*}$.

As an application of Theorem 1, the following partial solution to a similar problem will be provided.

THEOREM 3. Suppose $G$ is a finitely generated group, generated by subnormal subgroups $H$ and $K$. Let $A, B$ be normal subgroups of $H, K$ respectively such that $H / A, K / B$ satisfy max-sn (the maximal condition for subnormal subgroups). Then $J=\langle A, B\rangle$ is subnormal in $G$.

Glasgow Math. J. 25 (1984) 103-105. 
This work is an extract from the author's Ph.D. thesis: Subnormality in infinite groups (University of Wales, 1982). Gratitude is expressed to University College, Cardiff, for a Faculty of Science Bursary award.

2. Proofs. The main ingredient in the proof of Theorem 1 is Lemma 1 below, the first part of which is Theorem B of [4], while the second part may be deduced from this result in the same way as "Corollary B1" is deduced from "Theorem B" in [8]. (The permutizer of $K$ in $H$, denoted by $P_{H}(K)$, is the largest subgroup $P$ of $H$ such that $P K=K P$.)

LEMMA 1. Let $G$ be a group, generated by subnormal subgroups $H$ and $K$, such that $G / G^{\prime}$ has finite rank. Then, given integers $a, b$, there exists an integer $c$ such that $\gamma_{c}(G) \leq \gamma_{a}(H) \gamma_{b}(K)$. Also, for some integer $d, \gamma_{d}(H) \leq P_{H}(K)$.

We shall also require a further lemma.

Lemma 2. Suppose $H, K$ are subnormal in $G=H K$, and that $A, B$ are subgroups of finite index in $H, K$ respectively. Then $\langle A, B\rangle$ has finite index in $G$.

Actually this result remains true if the hypothesis of subnormality is removed, being a consequence of a theorem of B. H. Neumann on coverings by finitely many cosets (see Amberg [1, Lemma 5.1]). However, it may be quite easily proved by induction on the defect of $H$ in $G$, thus avoiding appeal to the more general result. The proof is omitted.

Finally, we require a lemma which is no doubt well-known and which may be seen to be the "nilpotent case" of Theorem 1.

Lemma 3. Suppose $G=\langle H, K\rangle$ is a nilpotent group of finite rank and that $A, B$ are (normal) subgroups of finite index in $H, K$ respectively. Then $J=\langle A, B\rangle$ has finite index in $G$.

Proof. We proceed by induction on $c$, the nilpotency class of $G$. We may clearly suppose $c \geq 2$ and that the appropriate inductive hypothesis holds. Then $J \gamma_{c}(G)$ has finite index in $G$, and it is enough to show that $\left|\gamma_{c}(G): J \cap \gamma_{c}(G)\right|$ is finite. Now, for some integer $d, x^{d} \in J$ for all $x \in H \cup K$. Further, $\gamma_{c}(G)$ is generated by commutators of the form $\left[x_{1}, \ldots, x_{c}\right]$, where the $x_{i}$ belong to $H \cup K$. Writing $y_{i}=x_{i}^{d}$, a simple inductive argument shows that, for each integer $j \leq c,\left[x_{1}, \ldots, x_{j}\right]^{d i} \equiv\left[y_{1}, \ldots, y_{i}\right]$ modulo $\gamma_{j+1}(G)$. Thus $\gamma_{c}(G) / \gamma_{c}(G) \cap J$ has exponent at most $d^{c}$ and is therefore finite, as required.

Proof of Theorem 1. Let $P=P_{H}(K)$. Then $A \cap P$ has finite index in $P$, which is subnormal in $G[9$, Lemma 3] and hence in $P K$. By Lemma 2, $\langle A \cap P, B\rangle$ has finite index in $P K$. Thus $|P K: J \cap P K|$ is also finite. By Lemma 1, there is an integer a such that $\gamma_{a}(H) \leq P$ and therefore an integer $c$ such that $\gamma_{c}(G) \leq P K$. Thus $\left|\gamma_{c}(G): J \cap \gamma_{c}(G)\right|$ is finite. Since $G / G^{\prime}$ has finite rank, so has $G / \gamma_{c}(G)$ [6]. Factoring by $\gamma_{c}(G)$, we may apply Lemma 3 to obtain the desired result. 
Proof of Theorem 3. We first reduce to the case where $H / A$ and $K / B$ are soluble. Let $P=P_{K}(A)$. Then $P$ normalises $B$ and permutes with $A$, and so $B^{A}$ is normal in $\langle A, B, P\rangle$. $J=B^{A} A$ is thus subnormal in $\langle A, B, P\rangle$. By Corollary $B .1$ of $[8]$, we know that there is an integer $\lambda$ such that $K^{(\lambda)} \leq P$, and so $J$ is subnormal in $\left\langle A, B K^{(\lambda)}\right\rangle$. Hence we may replace $B$ by $B K^{(\lambda)}$, i.e. we may suppose $K / B$ is soluble. Similarly $H / A$ may be assumed soluble. Since they satisfy max-sn, $H / A$ and $K / B$ are thus polycyclic, and it follows from a well-known theorem of Mal'cev (see e.g. Theorem 3.25 of [7]) that they are nilpotent-byabelian-by-finite. Let $L, M$ be normal subgroups of finite index in $H, K$ such that $(L / A)^{\prime}$, $(M / B)^{\prime}$ are nilpotent of class $a, b$ respectively. By Theorem 1 we may suppose $L=H$ and $M=K$, since $\langle L, M\rangle$ is also finitely generated and subnormal in $G$. Now, by Lemma 1 , there is an integer $c$ such that $\gamma_{c}(G) \leq H^{\prime} K^{\prime}$. So $\gamma_{c}(G) K^{\prime}=\left(\gamma_{c}(G) K^{\prime} \cap H^{\prime}\right) K^{\prime}=R K^{\prime}$, say, a product of two subnormal subgroups. We may thus apply Lemma 2 of [10] to deduce that, for some integer $d, \gamma_{d}\left(\gamma_{c}(G) K^{\prime}\right) \leq \gamma_{a+1}(R) \gamma_{b+1}\left(K^{\prime}\right) \leq A B$. Hence $\gamma_{d}\left(\gamma_{c}(G)\right) \leq J$ and, factoring, we may suppose that $G$ is metanilpotent. But in a finitely generated metanilpotent group, the join of any two subnormal subgroups is subnormal [5, Corollary 1 to Theorem 5.2] and so the theorem is proved.

Remark. The conclusion that $J$ is subnormal in $G$ also follows if "max-sn" is replaced by "min-sn" in Theorem 3. For, again reducing to the soluble case, we may this time assume that $H / A$ and $K / B$ are Černikov groups and hence abelian-by-finite.

\section{REFERENCES}

1. B. Amberg, Artinian and Noetherian factorized groups, Rend. Sem. Mat. Univ. Padova 55 (1976), 105-122.

2. G. Baumslag, Wreath products and p-groups, Proc. Cambridge Philos. Soc. 55 (1959), 224-231.

3. J. C. Lennox, D. Segal and S. E. Stonehewer, The lower central series of a join of subnormal subgroups, Math. Z. 154 (1977), 85-89.

4. J. C. Lennox and S. E. Stonehewer, The join of two subnormal subgroups, J. London Math. Soc. (2) 22 (1980), 460-466.

5. D. J. S. Robinson, Joins of subnormal subgroups, Illinois J. Math. 9 (1965), 144-168.

6. D. J. S. Robinson, A property of the lower central series of a group, Math. Z. 107 (1968), 225-231.

7. D. J. S. Robinson, Finiteness conditions and generalised soluble groups, Vol. 1 (Springer, 1972).

8. J. E. Roseblade, The derived series of a join of subnormal subgroups, Math. Z. 117 (1970), 57-69.

9. J. E. Roseblade and S. E. Stonehewer, Subjunctive and locally coalescent classes of groups, J. Algebra 8 (1968), 423-435. 45-54.

10. S. E. Stonehewer, Nilpotent residuals of subnormal subgroups, Math. Z. 139 (1974),

11. H. Wielandt, Eine Verallgemeinerung der invarienten Untergruppen, Math. Z. 45 (1939), 209-244.

\section{Department of Pure Mathematics \\ UNIVERSITY COLlege \\ CARDIFF}

